Towards resolving the complex paramagnetic NMR spectrum of small laccase: Assignments of resonances to residue specific nuclei

Rubin Dasgupta, Karthick B.S.S. Gupta, Huub J.M. de Groot, Marcellus Ubbink*

Leiden Institute of Chemistry, University of Leiden, Gorlaeus Laboratory, Einsteinweg 55, 2333 CC, Leiden, The Netherlands.

Correspondence to: Marcellus Ubbink (m.ubbink@chem.leidenuniv.nl) 


\section{Protein expression and purification}

SLAC-T1D and SLAC-T1D/Y108F were expressed and purified as described previously (Dasgupta et al., 2020; Machczynski et al., 2004). For ${ }^{15} \mathrm{~N}$ histidine specific perdeuterated labelled sample, $50 \mathrm{mg} / \mathrm{L}$ of ${ }^{15} \mathrm{~N}_{3}$-L-histidine hydrochloride monohydrate (Sigma Aldrich, USA) was added to the M9 medium consisting of ammonium chloride and D-glucose-1,2,3,4,5,6,6-d7 as nitrogen and carbon sources respectively. A volume of $200 \mu \mathrm{L}$ of the $\mathrm{M} 9$ preculture was transferred to $25 \mathrm{~mL}$ of $\mathrm{M} 9$ medium prepared in $99.99 \% \mathrm{D}_{2} \mathrm{O}$ for an overnight preculture, which was used to inoculate $500 \mathrm{~mL} \mathrm{D}_{2} \mathrm{O}-\mathrm{M} 9$ minimal medium. Gene expression and protein harvesting was done as for the uniform ${ }^{15} \mathrm{~N}$ labelled sample. Purity was checked by SDS PAGE using a precast Bis-Tris gel (ThermoFischer scientific), as shown in Figure S1a. A band $\sim 74 \mathrm{kDa}$ is observed. Under native condition from size exclusion chromatography with multi-angle light scattering (SEC-MALS) the molecular weight of the proteins was $\sim 105 \mathrm{kDa}$, in accord with the expected trimeric form.

\section{NMR spectroscopy}

Samples contained $\sim 1 \mathrm{mM}$ of protein in $10 \mathrm{mM}$ sodium phosphate buffer $\mathrm{pH} 7.3$ with $10 \% \mathrm{D}_{2} \mathrm{O}$. Experiments were done on a Bruker AV-III HD $600 \mathrm{MHz}$ NMR spectrometer equipped with a TXI cryoprobe. $1 \mathrm{D}^{1} \mathrm{H}$ WEFT, Inversion recovery experiments to measure the spin-lattice relaxation rate and 2D ${ }^{1} \mathrm{H}-{ }^{1} \mathrm{H}$ EXSY/NOESY (Jeener et al., 1979) experiments were recorded as described previously (Dasgupta et al., 2020). The mixing time dependent integral volume profiles were fitted using equation described previously (Dasgupta et al., 2020; Farrow et al., 1994) with Igor Pro 6.3.7 to obtain the exchange rates. The fitting was done by constraining the $\mathrm{K}_{\text {eq }}$ (Table $\mathrm{S} 1$ ) to the value of the ratio of the diagonal integral volume at $0 \mathrm{~ms}$ mixing time. The spin-lattice relaxation rates used in the fitting were obtained from inversion recovery experiments.

$2 \mathrm{D}{ }^{1} \mathrm{H}-{ }^{15} \mathrm{~N} \mathrm{HMQC}$ experiments were recorded using the pulse sequence shown in Figure S2. The transfer delay d2 was optimized to $0.5 \mathrm{~ms}$ to enhance the paramagnetically shifted signals $>20$ ppm. Forty eight $\mathrm{t} 1$ increment points were acquired with 30720 number of scans corresponding to the total acquisition time of $54 \mathrm{~h}$ for each experiment. 
Equation to fit the temperature dependence of the chemical shift for the two-metal center

The observed chemical shift $\delta_{o b s}$ is given by

$$
\delta_{o b s}=\delta_{d i a}+\frac{A}{\hbar} \frac{g_{e} \mu_{B}}{\gamma k_{B} T}\left[\frac{e^{\left(\frac{2 J}{k_{B} T}\right)}}{1+3 e^{\left(\frac{2 J}{k_{B} T}\right)}}\right]
$$

where $\delta_{d i a}$ is the diamagnetic chemical shift, $A$ is the isotropic hyperfine coupling constant, $g_{e}$ is the free-spin electron $g$ factor with the value of $2.0023, \mu_{B}$ is the electron Bohr magneton, $\hbar$ is the reduced Planck constant, $\gamma$ is the gyromagnetic ratio of the nucleus in question, $k_{B}$ is the Boltzmann constant, $T$ is the temperature in Kelvin and $S=1 / 2$ which is the spin of the $\mathrm{Cu}(\mathrm{II})$ ion (Bertini et al., 2017; Machczynski et al., 2004). 


\section{Supporting Figures}

(a)

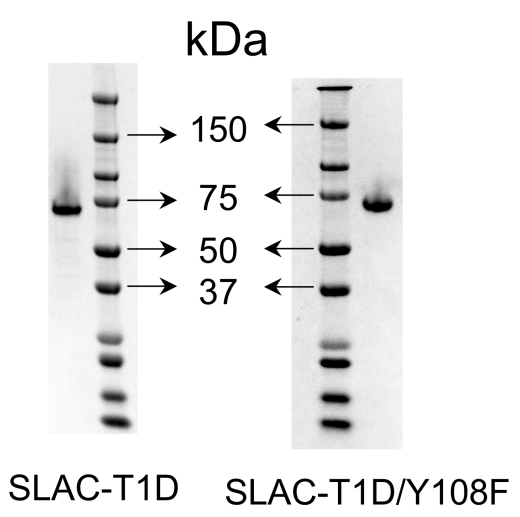

(b)

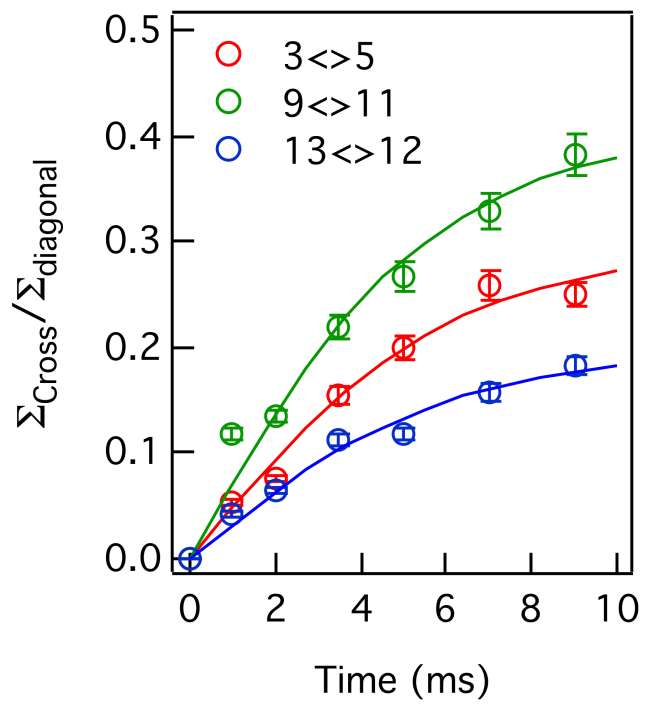

Figure S1: (a) Image of a Bis-Tris precast SDS PAGE gel (ThermoFischer scientific) of purified SLACT1D and SLAC-T1D/Y108F; (b) Fits of the intensity profile of normalized cross peak integrals from the resonance pairs of 3-5, 9-11 and 13-12 from 2D ${ }^{1} \mathrm{H}-{ }^{1} \mathrm{H}$ EXSY of SLAC-T1D/Y108F to determine the exchange rates (Dasgupta et al., 2020; Farrow et al., 1994). The error bars represent the standard error of the mean derived from the duplicate experiments. 


\section{${ }^{1} \mathrm{H}-{ }^{15} \mathrm{~N} H M Q C$}

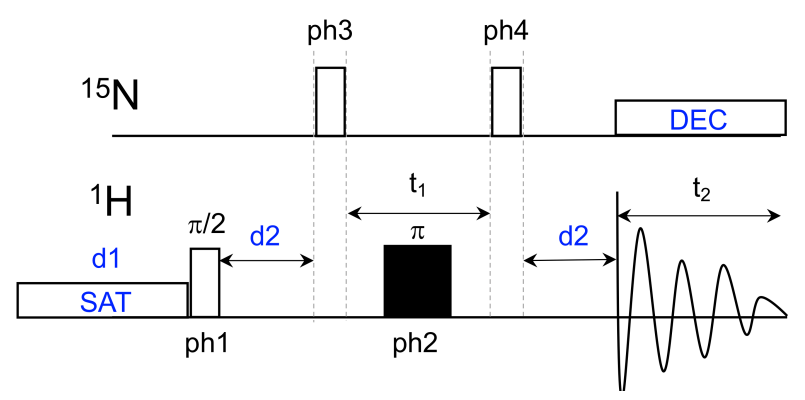

Figure S2: ${ }^{1} \mathrm{H}-{ }^{15} \mathrm{~N}$ HMQC pulse sequence used in this study. Open rectangles are for $90^{\circ}$ pulse and filled ones are for $180^{\circ}$ pulse. The interscan delay d 1 is $100 \mathrm{~ms}$ and SAT is the continuous wave water saturation pulse with phase of $x$. DEC is GARP composite pulse decoupling during the $t_{2}$ evolution. The evolution period d2 was set to $0.5 \mathrm{~ms}$, corresponding to the FWHM of the paramagnetically shifted ${ }^{1} \mathrm{H}$ resonances ( $0.8 \mathrm{ppm})$ (Bertini et al., 2017; Ciofi-Baffoni et al., 2014). The phase cycling is ph1 =x, $\mathrm{ph} 2=\mathrm{x}, \mathrm{ph} 3=\mathrm{x}-\mathrm{x}, \mathrm{ph} 4=\mathrm{x} \mathrm{x}-\mathrm{x}-\mathrm{x}$ and for the receiver it was $\mathrm{x}-\mathrm{x} \mathrm{x}-\mathrm{x}$. 
(a)

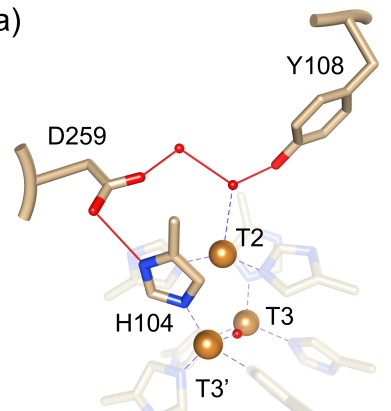

(b)

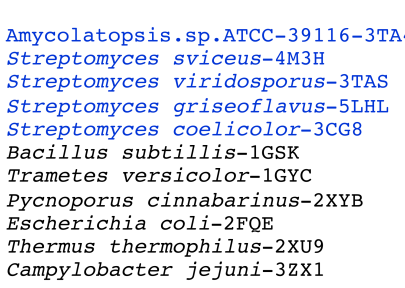

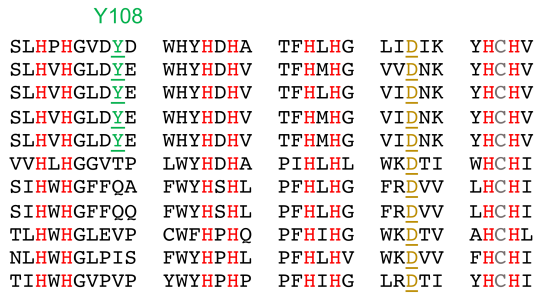

D259

Figure S3. The second coordination shell residue Tyr108. (a) Crystal structure of SLAC (PDB entry 3cg8, resolution $2.63 \AA$ ) (Skálová et al., 2009), showing the hydrogen bonding network between Tyr108 and His104 via Asp259 (red lines). The hydrogen bonds were determined by the default parameter of UCSF Chimera program (Pettersen et al., 2004) with relaxed hydrogen bond constraints of $0.4 \AA$ and $20.0^{\circ}$ (Mills and Dean, 1996); (b) Sequence alignment of the two-domain (blue) and three-domain laccases (black) for which crystal structures are available (PDB code indicated). The copper coordinating residues are shown in red. Tyr108 and Asp259 (numbering from PDB 3cg8) are shown in green and gold, respectively and are underlined. The type 1 copper site ligand, cysteine, is marked in grey. 
(a)

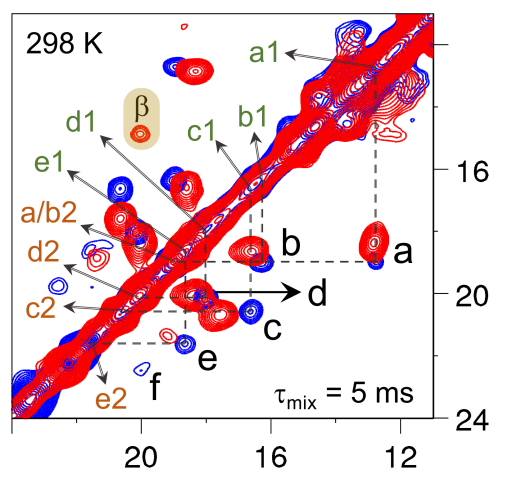

(b)

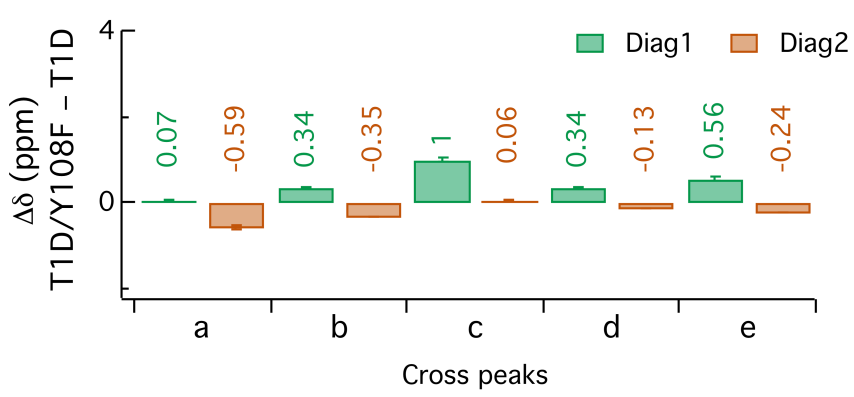

SLAC-T1D SLAC-T1D/Y108F

Figure S4. Comparison of the RO spectral regions for SLAC-T1D and SLAC-T1D/Y108F. (a) Overlayed ${ }^{1} \mathrm{H}-{ }^{1} \mathrm{H}$ EXSY/NOESY spectra at $298 \mathrm{~K}$ with mixing time of $5 \mathrm{~ms}$ of SLAC-T1D (blue) and SLACT1D/Y108F (red) for the region between 11 and 22 ppm. Cross-peaks and diagonal peaks for SLACT1D are marked. The new cross peak $\beta$ in SLAC-T1D/Y108F is highlighted; (b) Chemical shift changes between SLAC-T1D and SLAC-T1D/Y108F. Positive values mean the diagonal peak in SLACT1D/Y108F is downfield shifted, while for the negative value it is upfield shifted. 

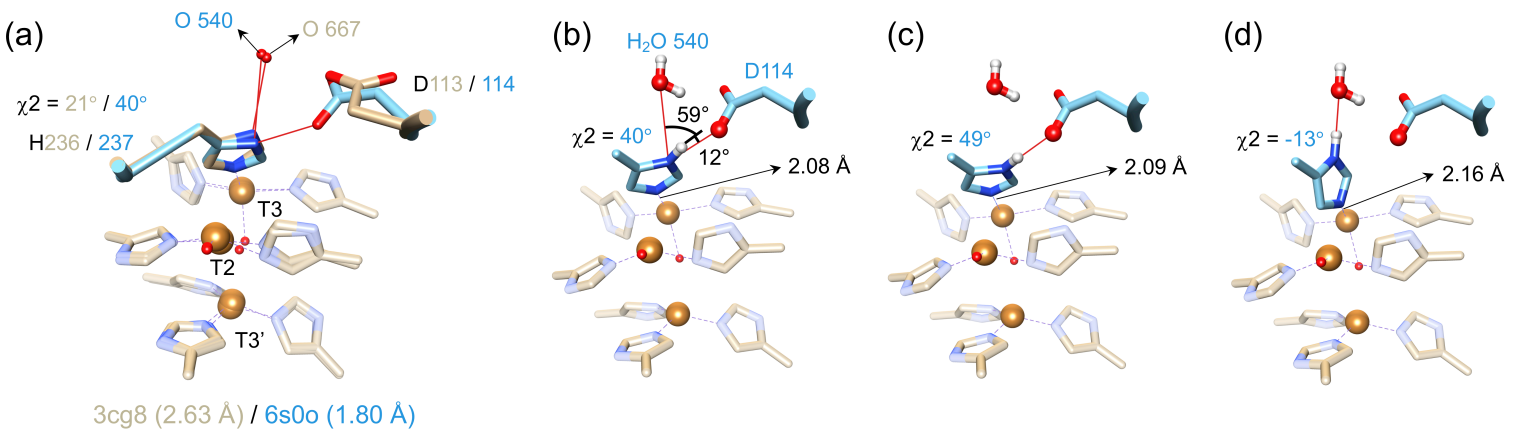

Figure S5. Two H-bond acceptors for His236. (a) Overlayed TNC from crystal structure 3cg8 in gold and $6 \mathrm{~s} 0 \mathrm{o}$ in blue highlighting the hydrogen bond of the $\mathrm{N} \delta 1$ from the T3 histidine ligand His236/237 (Gabdulkhakov et al., 2019; Skálová et al., 2009). The $\chi 2$ dihedral angle for histidine 236/237 is shown for both crystal structures. Hydrogen bonds are shown as red lines; (b) Protons are modelled for the crystal structure 6s0o using the algorithm as implemented in UCSF Chimera (Gabdulkhakov et al., 2019; Pettersen et al., 2004). The values of the angles between [Asp114 O 11 - His237 N $\delta 1$ - His237 $\mathrm{H} \delta 1$ ] and [water O540 - His237 N81 - His237 H81] are indicated along with the distance between His237 Nع2 - T3 copper (with arrows); (c, d) The ring rotation that brings the $\mathrm{H} \delta 1$ to the optimal position for a hydrogen bond with Asp114 O 81 (c) and the water (d) are shown. The new $\chi 2$ dihedral angle and the corresponding His237 Nع2 - T3 copper distances are indicated. 
Table S1. Exchange and spin lattice relaxation rates at $298 \mathrm{~K}$ for SLAC-T1D/Y108F. In brackets the values for SLAC-T1D are shown for comparison (Dasgupta et al., 2020). Errors in the values are $\sim 5 \%$ from the duplicate experiments.

\begin{tabular}{cccc}
\hline & $3-5$ & $9-11$ & $13-12$ \\
\hline $\mathrm{k}_{\mathrm{A}}\left(\mathrm{s}^{-1}\right)$ & $37(33)$ & $51(29)$ & $26(29)$ \\
$\mathrm{k}_{\mathrm{B}}\left(\mathrm{s}^{-1}\right)$ & $60(62)$ & $91(91)$ & $39(34)$ \\
$\mathrm{k}_{\mathrm{ex}}\left(\mathrm{s}^{-1}\right)$ & $97(95)$ & $142(120)$ & $65(63)$ \\
$\mathrm{K}_{\text {eq }}\left(\mathrm{k}_{\mathrm{A}} / \mathrm{k}_{\mathrm{B}}\right)$ & $0.62(0.53)$ & $0.56(0.32)$ & $0.67(0.85)$ \\
$\mathrm{R} 1_{\mathrm{A}}\left(\mathrm{s}^{-1}\right)$ & $225(225)$ & $240(240)$ & $170(170)$ \\
$\mathrm{R} 1_{\mathrm{B}}\left(\mathrm{s}^{-1}\right)$ & $518(457)$ & $524(464)$ & $483(492)$ \\
\hline
\end{tabular}


Table S2. ${ }^{1} \mathrm{H}$ and ${ }^{15} \mathrm{~N}$ chemical shift in ppm of the paramagnetically shifted resonances $(>21 \mathrm{ppm}$ in 1D ${ }^{1} \mathrm{H}$ WEFT spectrum) at $298 \mathrm{~K}$. Resonance showing no change between SLAC-T1D/Y108F and SLAC-T1D are highlighted. Resonance pairs undergoing chemical exchange are color coded as red for $3-5$, green for 9-11 and blue for $13-12 .{ }^{15} \mathrm{~N}$ chemical shift of resonance $7,8,17$ and 18 are not observed from the ${ }^{1} \mathrm{H}-{ }^{15} \mathrm{~N} \mathrm{HMQC}$ spectra and are highlighted in grey. Resonance 7 and 8 were reported to be carbon attached protons (Dasgupta et al., 2020) while the ${ }^{1} \mathrm{H}$ resonance of 17 and 18 are broad ( 1.1 ppm), which can affect the evolution period d2 in the ${ }^{1} \mathrm{H}-{ }^{15} \mathrm{~N} \mathrm{HMQC}$ (Figure S2). Since the $\mathrm{d} 2$ was set to $0.5 \mathrm{~ms}$, corresponding to the ${ }^{1} \mathrm{H}$ line with of $0.8 \mathrm{ppm}$ at $600 \mathrm{MHz}$, resonance 17 and 18 might not be observable due to their larger line broadening.

\begin{tabular}{ccc|cc} 
Resonance & \multicolumn{2}{c}{ SLAC-T1D } & \multicolumn{2}{c}{ SLAC-T1D/Y108F } \\
\hline & ${ }^{1} \mathrm{H}$ & ${ }^{15} \mathrm{~N}$ & ${ }^{1} \mathrm{H}$ & ${ }^{15} \mathrm{~N}$ \\
\hline 3 & 22.00 & 291.65 & 22.24 & 294.24 \\
4 & 23.40 & 245.21 & 24.53 & 251.16 \\
5 & 24.75 & 330.35 & 25.07 & 333.41 \\
6 & 26.46 & 461.91 & 25.95 & 459.73 \\
7 & 29.26 & - & 29.26 & - \\
8 & 31.65 & - & 31.65 & - \\
9 & 34.88 & 450.30 & 35.43 & 456.79 \\
11 & 36.95 & 477.39 & 37.68 & 485.19 \\
12 & 39.24 & 535.44 & 39.20 & 539.05 \\
13 & 41.82 & 581.87 & 42.04 & 565.49 \\
15 & 43.09 & 569.04 & 43.13 & 570.38 \\
16 & 44.11 & 574.13 & 43.67 & 559.61 \\
17 & 49.17 & - & 49.17 & - \\
18 & 52.52 & - & 52.52 & - \\
\hline
\end{tabular}


Table S3. ${ }^{1} \mathrm{H}$ and ${ }^{15} \mathrm{~N}$ chemical shift in ppm at $298 \mathrm{~K}$ for the resonance between 12 and $21 \mathrm{ppm}$ of the RO state.

\begin{tabular}{ccc} 
Resonance & \multicolumn{2}{c}{ SLAC-T1D } \\
\hline & ${ }^{1} \mathrm{H}$ & ${ }^{15} \mathrm{~N}$ \\
\hline $\mathrm{a} 1$ & 12.82 & 179 \\
$\mathrm{a} 2$ & 19.01 & 266 \\
$\mathrm{~b} 1$ & 16.29 & - \\
$\mathrm{b} 2$ & 19.06 & 270 \\
$\mathrm{c} 1$ & 16.65 & 248 \\
$\mathrm{c} 2$ & 20.59 & 277 \\
$\mathrm{~d} 1$ & 18.01 & - \\
$\mathrm{d} 2$ & 20.02 & 275 \\
$\mathrm{e} 1$ & 18.64 & - \\
$\mathrm{e} 2$ & 21.64 & - \\
$\mathrm{x} 1$ & 13.58 & 185 \\
$\mathrm{x} 2$ & 14.68 & 213 \\
$\mathrm{y}$ & 15.43 & 232 \\
$\mathrm{w}$ & 13.29 & 190 \\
$\mathrm{z}$ & 19.90 & 265 \\
\hline
\end{tabular}


Table S4. Potential hydrogen bond acceptors for T3 His N $\delta 1$ atoms as defined by (Mills and Dean, 1996). Data based on chains $E$ and F from PDB entry $6 s 00$ (resolution 1.80Å) (Gabdulkhakov et al., 2019).

\begin{tabular}{|c|c|}
\hline Histidine & $\begin{array}{c}\text { Potential hydrogen bond } \\
\text { acceptors }\end{array}$ \\
\hline His 105.E & Asp 260.F O $\delta 1$ and $\mathrm{H}_{2} \mathrm{O} 546 . \mathrm{E} \mathrm{O}$ \\
\hline His 290.F & Gln 292.F O $\varepsilon 1$ and $\mathrm{H}_{2} \mathrm{O} 540 . \mathrm{F} \mathrm{O}$ \\
\hline His 159.E & Glu 164.E CO and $\mathrm{H}_{2} \mathrm{O}$ 540.E O \\
\hline His $237 . F$ & Asp 114.E O 81 and $\mathrm{H}_{2} \mathrm{O}$ 501.E O \\
\hline
\end{tabular}




\section{Supporting References}

Bertini, I., Luchinat, C., Parigi, G. and Ravera, E.: NMR of paramagnetic molecules: applications to metallobiomolecules and models, Second edition., Elsevier, Amsterdam., 2017.

Ciofi-Baffoni, S., Gallo, A., Muzzioli, R. and Piccioli, M.: The IR- ${ }^{15} \mathrm{~N}-H S Q C-A P$ experiment: a new tool for NMR spectroscopy of paramagnetic molecules, Journal of Biomolecular NMR, 58(2), 123-128, doi:10.1007/s10858-013-9810-2, 2014.

Dasgupta, R., Gupta, K. B. S. S., Nami, F., Groot, H. J. M. de, Canters, G. W., Groenen, E. J. J. and Ubbink, M.: Chemical Exchange at the Trinuclear Copper Center of Small Laccase from Streptomyces coelicolor, Biophysical Journal, 119(1), 9-14, doi:10.1016/j.bpj.2020.05.022, 2020.

Farrow, N. A., Zhang, O., Forman-Kay, J. D. and Kay, L. E.: A heteronuclear correlation experiment for simultaneous determination of ${ }^{15} \mathrm{~N}$ longitudinal decay and chemical exchange rates of systems in slow equilibrium, J Biomol NMR, 4(5), 727-734, doi:10.1007/BF00404280, 1994.

Gabdulkhakov, A., Kolyadenko, I., Kostareva, O., Mikhaylina, A., Oliveira, P., Tamagnini, P., Lisov, A. and Tishchenko, S.: Investigations of Accessibility of T2/T3 Copper Center of Two-Domain Laccase from Streptomyces griseoflavus Ac-993, International Journal of Molecular Sciences, 20(13), 3184, doi:10.3390/ijms20133184, 2019.

Jeener, J., Meier, B. H., Bachmann, P. and Ernst, R. R.: Investigation of exchange processes by twodimensional NMR spectroscopy, J. Chem. Phys., 71(11), 4546-4553, doi:10.1063/1.438208, 1979.

Machczynski, M. C., Vijgenboom, E., Samyn, B. and Canters, G. W.: Characterization of SLAC: A small laccase from Streptomyces coelicolor with unprecedented activity, Protein Science, 13(9), 2388-2397, doi:10.1110/ps.04759104, 2004.

Mills, J. E. J. and Dean, P. M.: Three-dimensional hydrogen-bond geometry and probability information from a crystal survey, J Computer-Aided Mol Des, 10(6), 607-622, doi:10.1007/BF00134183, 1996.

Pettersen, E. F., Goddard, T. D., Huang, C. C., Couch, G. S., Greenblatt, D. M., Meng, E. C. and Ferrin, T. E.: UCSF Chimera-A visualization system for exploratory research and analysis, Journal of Computational Chemistry, 25(13), 1605-1612, doi:10.1002/jcc.20084, 2004.

Skálová, T., Dohnálek, J., Østergaard, L. H., Østergaard, P. R., Kolenko, P., Dušková, J., Štěpánková, A. and Hašek, J.: The Structure of the Small Laccase from Streptomyces coelicolor Reveals a Link between Laccases and Nitrite Reductases, Journal of Molecular Biology, 385(4), 1165-1178, doi:10.1016/j.jmb.2008.11.024, 2009. 